\title{
Nebivolol: An Appealing, Awaited and Nitric Oxide Potentiator Drug for the Treatment of Heart Failure
}

\author{
Aryendu Kumar Saini'", Pranay Wal', Manisha Verma², Suresh Chandra', Anurag Singh', Sanjeev Yadav', Himanshu Bansode', \\ Kumar Nischaya ${ }^{1}$, Nikita Saraswat ${ }^{1}$ \\ ${ }^{1}$ Pharmacy Department, Pranveer Singh Institute of Technology, Kanpur, Uttar Pradesh, INDIA \\ ${ }^{2}$ Department of Clinical Research, National Institute of Pharmaceutical Education and Research, Mohali, Punjab, INDIA.
}

\begin{abstract}
Nebivolol is a third generation beta-blocker that possesses the highest selectivity for beta- 1 receptors than beta- 2 receptors than any other beta blocker in its class. Long-term therapy of nebivolol, in clinical trials, has been found to be very effective in increasing the left ventricular ejection fraction, reducing all-cause mortality, and reducing the New York Heart Association (NYHA) class of heart failure. The novelty of nebivolol exists in its unique nitric oxide potentiating effects in the myocardial and endothelial cells by the activation of beta-3 adrenergic receptors by which it produces most of its actions. It produces many useful effects and helps to abate the disease progression and prolongs the survival. In many clinical studies, it has been found to be safe and well tolerated. A literature search was made on PubMed database with the keywords: "Heart failure", "Nebivolol", and "beta- blocker". A total of 176 articles were found and were the combination of review articles and research articles. A further selection of articles was made by the exclusion of non-relevant articles to achieve the objective of this review. It is approved for the treatment of hypertension in the United
\end{abstract}

States and is under review by the federal agency, United States Food and Drugs Administration for the treatment of heart failure. This article sole intention is to review all the clinically important aspects of nebivolol on the basis of which nebivolol can be definitely considered for its inclusion in the drug therapy list for heart failure which will ease the individualization of drug therapy.

Key words: Cardiac remodeling, Heart failure, Hypertrophy, Nebivolol, Nitric oxide, Systolic dysfunction.

Correspondence

Aryendu Kumar Saini, MS-958, Auri More, Anpara, Sonbhadra, Uttar Pradesh, INDIA.

Phone: 7800642030

Email: aryendu77@gmail.com

DOI: 10.5530/jyp.2018.10.34

\section{INTRODUCTION}

Heart failure is a complex clinical syndrome that is characterized by the inefficiency of the heart muscle to pump or fill with the blood. This like other syndromes has become a major killer of mankind. Our body requires a continuous supply of blood to meet the body's needs. ${ }^{1}$ However, the heart is not able to keep its workload in the heart failure condition. This clinical syndrome is mostly seen in the people of age 65 or more and is related to the mortality and morbidity. ${ }^{2}$ The problem arises most often in the left ventricle because it is the left ventricle that acts as main pumping chamber and pumps the oxygenated blood all over the body. This generally becomes the reason of its impairment than any other chambers of the heart, and this failure in which the left ventricle functions get impaired is called as left-sided heart failure or left ventricular heart failure. To evaluate pumping function of the left ventricle, ejection fraction is the most common parameter that is measured. ${ }^{3}$ Ejection fraction is measured in percentage and refers to the percentage of the blood that leaves the ventricle each time the heart contracts. Ejection fraction is conventionally measured for the left ventricles and this is the reason why in most cases, Ejection Fraction commonly refers to Left Ventricular Ejection Fraction (LVEF). ${ }^{3,4}$ LV (Left Ventricle) ejection fraction of more than $50 \%$ is considered as normal. The heart failure on the premise of ejection fraction is divided into two types namely heart failure with reduced ejection fraction and the heart failure with preserved ejection fraction. In both the types, stroke volume is affected and gets reduced.
In patients suffering from heart failure with reduced ejection fraction (systolic dysfunction), contractility of left ventricles of heart gets affected while in the heart failure with preserved ejection (diastolic dysfunction), the stiffness of the left ventricular muscles takes place that affects the relaxing property of left ventricles. About $60 \%$ of patients suffer from the systolic dysfunction that occurs as a result of end-stage coronary artery disease. $^{5}$

Nebivolol, being a third generation beta-blocker, selectively antagonizes the beta-1 receptors present on the heart. ${ }^{6}$ It is very highly selective beta-1 antagonist known to date and is unique in its mechanism of action that makes it apart from rest of the members of its class like carvedilol, bisoprolol, etc. Nebivolol apart from protecting the heart from long-term effects of catecholamines activates the beta- 3 receptors present on the endothelial cells and myocardial cells, and by activating these receptors; it mediates the production of nitric oxide from the L-arginine-nitric oxide pathway. The major actions are produced via the nitric oxide which itself is a wonderful molecule. ${ }^{6,7}$ This molecule exerts some outstanding effects by reducing the hypertrophy, cardiac remodeling, decreasing the total peripheral resistance and preload, etc. The clinical studies demonstrate beneficial effects in systolic heart failure suggesting increased ejection fraction and improved New York Heart Association (NYHA) class. The NYHA classification of heart failure is given in Table 1. 
Saini, et al:: Nebivolol: An Appealing, Awaited, and Nitric Oxide Potentiator Drug for the Treatment of Heart Failure

Table 1: NYHA Classification showing classes and its description based on limitations on the physical activity of patients.

\begin{tabular}{cc}
\hline NEW YORK HEART ASSOCIATION CLASSIFICATION (NYHA) OF HEART \\
FAILURE
\end{tabular}

The adverse effects profile found in the clinical trials suggest the good tolerability and patient safety assurance. ${ }^{8}$ Nebivolol has been proved that it reduces the all-cause mortality, and morbidity in clinical trials but currently, Nebivolol is not a United States Food and Drugs Administration (US FDA) approved drug for the treatment of heart failure and despite exhibiting excellent therapeutic effects in heart failure found in most of the clinical studies, most important reason for its lack of acceptance by the US FDA is the bias in the trial study named "Study of the Effects of Nebivolol Intervention on Outcomes and Re-hospitalization in Seniors" (SENIORS) trial (the only trial that included the elder patients) ${ }^{9}$ and another important reason is that despite being a proven drug for its efficacy in systolic dysfunction, trials related to the effect of nebivolol in diastolic dysfunction are less. ${ }^{10,11}$ The main objective of this review is to encompass and provide clinical insights of all the features of nebivolol that are sufficient to anticipate that nebivolol would surely be a drug that will be used in parallel with other standard therapies if more clinical data become available in the future.

\section{MATERIALS AND METHODS}

A literature search was conducted on the PubMed database with the help of keywords: "Heart Failure", "Nebivolol" and "Beta-blocker". The search was customized by applying the filter so as to get only clinical studies (research articles) and review articles. The result showed a total number of 176 articles and articles which were complying and having relevance to the objective of this review were studied. Furthermore, the help of 2 websites namely "Medscape" and "Cleveland Clinic" was taken that were worth the source of information and helped to accomplish this review.

\section{CLINICAL PHARMACODYNAMICS OF NEBIVOLOL}

\section{Dynamic Roles of Nitric Oxide and Nebivolol as its Potentiator}

The blood vessels have an inner lining of endothelial cells that acts as a barrier between the smooth muscle and the circulating blood. These endothelial cells are important for maintaining the vascular tone, preventing the platelets aggregation, and inhibiting the proliferation of vascular smooth muscle cells. ${ }^{12}$ These all functions are mediated by a potent vasodilator molecule which is produced by the endothelial cells. This molecule is nothing but the Nitric Oxide (NO). ${ }^{13}$ The nitric oxide is produced by the endothelial cell via the $\mathrm{L}$-arginine-nitric oxide pathway. The substrate for its production is L-arginine which is an amino acid. This synthesis is catalyzed by an enzyme known as nitric oxide synthase. ${ }^{14}$ There are three isoforms of this enzyme namely endothelial nitric oxide synthase (found in the cardiomyocytes and endothelial cells), neuronal nitric oxide synthase (found in the brain), and inducible nitric oxide synthase (found in macrophages). In this pathway, nitric oxide synthase cleaves the nitrogen from the guanidino group of arginine and subsequently form citrulline and $\mathrm{NO}$.

This reaction needs the cofactors like tetrahydrobiopterin, FAD (Flavin Adenine Dinucleotide), NADPH (Nicotinamide Adenine Dinucleotide Phosphate), and FMN (Flavin Mononucleotide). ${ }^{15}$ There are sufficient findings to confirm the fact that Nebivolol by NO potentiation causes vasodilation. Example of one such study is that when intra-arterial infusion of Nebivolol was given, it caused vasodilatation and increased blood flow in the forearm which was not seen with atenolol and the vasorelaxant effect of nitric-oxide was antagonized by L-nitro-monomethyl arginine (L-NMMA), which is an inhibitor of NO synthase, indicates that nitric oxide is responsible for vasodilatation. ${ }^{16}$

Nebivolol has agonistic property at beta- 3 receptors which are present on the endothelium. When nebivolol agonizes the beta- 3 receptor, a conformational change in the receptor takes place which causes the alpha sub-unit of $\mathrm{G}_{\mathrm{s}}$ (G-Stimulatory) protein to acquire Guanosine Triphosphate (GTP) in place of Guanosine Diphosphate (GDP). The GTP bound alpha subunit then separates from the $\beta \gamma$ diamer part of $G_{s}$ protein and activates the adenylyl cyclase (AC) enzyme. The adenylyl cyclase enzyme converts the Adenosine Triphosphate (ATP) into cyclic Adenosine Monophosphate (cAMP). This cAMP in turn activates the Protein Kinase $\mathrm{A}\left(\mathrm{PK}_{\mathrm{A}}\right)$. This kinase phosphorylates and modifies the function of many enzymes like nitric-oxide synthase.

The production of nitric oxide by the NO synthase present in the endothelium is enhanced..$^{14,16}$ Moreover, in a study by Gauthier et al. it was found that beta- 3 adrenoreceptors are also present and functional in the heart and were responsible for the unexpected negative inotropic effects of epinephrine, norepinephrine, and was important in the pathophysiology of heart diseases and indeed, beta- 3 receptors found to be important in the pathophysiology of different cardiovascular diseases including heart failure. ${ }^{17}$ The effects of nebivolol actions on the heart are mediated via beta-3 receptors to release nitric oxide. Nebivolol confers the cardio protective effects that have proven to be beneficial for the treatment of heart failure. ${ }^{18}$

A useful attribute of $\mathrm{NO}$ is anti-atherogenic activity and hence, it prevents the coronary artery disease. The coronary artery disease can further lead to the deprivation of oxygen supply to the myocardial cell, thus, the death of the myocardial cell.

Nitric-oxide plays a very significant role in improving the efficiency of the left ventricle by decreasing the total peripheral resistance, which is not seen with many other traditional blockers like metoprolol and atenolol. Prevention of platelet aggregation (another useful attribute of NO), which is a central factor in the pathogenesis of unstable angina, can further lead to the obstruction of coronary arteries which can eventually lead to infarcts in the myocardium ${ }^{19}$ and thus loss of contractility (loss of myocardial cells), and heart failure. In chronic heart failure, due to different factors like increased preload (end-diastolic volume); ventricular hypertrophy (increase in the LV mass) and cardiac remodeling occurs as a chronic compensatory mechanism.

Cardiac remodeling or ventricular remodeling refers to a broader term indicating changes in both the extracellular matrix and the cardiomyocytes. ${ }^{20}$ This remodeling involves changes in the shape, size, and function of heart that can ultimately result in further destruction of systolic and ventricular function, thus causing myocardial injury and premature death. It has been found in the studies that nitric oxide is an important regulator of ventricular hypertrophy and cardiac remodeling. Nitric oxide by activating the guanylate cyclase increases the level of cyclic GMP 
(cGMP), and cGMP through protein kinase G inhibits the signaling networks of diverse pro-hypertrophic pathways that lead to ventricular hypertrophy.

In a study conducted by Wollert et al. it was found that transient release of nitric oxide exerts very beneficial effects in the remodeling process by decreasing the cavity dilation, hypertrophy, and mortality. ${ }^{21,22}$ All these findings demonstrate the anti-hypertrophic property of nitric oxide potentiated by nebivolol which is of utmost importance in the treatment of heart failure.

A study by Aragon et al. states that nebivolol is very effective in protecting against the myocardial ischemia-reperfusion injury by activating the neuronal and endothelial nitric oxide synthase and thus increasing the nitric oxide bioavailability. ${ }^{23}$ Another study by Wink et al. has demonstrated that nitric oxide modulates the cellular as well as physiological processes, protects from cell death, and reduces the oxidative injury moderated by alkyl-hydro peroxides, hydrogen peroxides, and xanthine oxidases. ${ }^{24}$ These studies are sufficient to present concrete shreds of evidence that nebivolol by potentiating and increasing the bioavailability of nitric oxide exerts beneficial effects in heart failure.

Another condition, hypertension, is associated with abnormal nitric oxide production and bioavailability. In many chronic hypertensive patients, endothelial dysfunction (which causes less synthesis of NO), has been noted. In chronic hypertensive conditions, the heart has to work harder to push the blood. So, in order to cope up with this situation, the heart becomes stiffer and thicker, which tends to cause it less able to pump effectively.

This eventually leads to heart failure. Nebivolol by mediating the L-arginine-NO pathway helps to increase the synthesis of nitric oxide and also works as a prophylactic factor. So, nebivolol by mediating the pathway of formation of nitric oxide increases its bioavailability and exerts magnificent effects. ${ }^{25}$

\section{Neuro-hormonal activation and actions of Nebivolol}

Most of the patients with heart failure suffer from dysfunction or the impairment of left ventricles, that is manifested as a reduction in the stroke volume and hence the cardiac output. This leads to the activation of compensatory mechanisms to overcome low cardiac output. Neurohormonal compensation is one of the early mechanism by which heart tries to maintain the blood supply to the body. It includes the activation of the sympathetic nervous system and rennin-angiotensin-aldosterone system (RAAS). The sympathetic nervous system gets activated by high and low-pressure baroreceptors as an early compensatory mechanism in order to maintain and augment cardiac output.

The epinephrine and nor-epinephrine increases the ionotrophy (force of contraction), chronograph (rate of contraction) and atrio-ventricular node conduction. ${ }^{26}$ Moreover, nor-epinephrine activates the beta- 1 receptors present on the juxtaglomerular cells, which releases renin. Renin is responsible for the activation of RAAS. The consequences of these two activated systems will be finally increased preload, afterload, and vasoconstriction. Although, this neuro-hormonal compensatory mechanism acts as a good support for the heart under the normal physiological conditions but chronic activation exerts deleterious effects on the heart. Chronic RAAS activation leads to fibrosis, myocardial cell apoptosis, and cardiac remodeling, and endothelial dysfunction.

If ventricles are chronically stressed by increased preload, it leads to structural changes in the heart, which is left ventricular hypertrophy $(\mathrm{LVH})$. It is the thickening and the enlargement of the left ventricle in response to increased preload. This enlarged ventricular muscle loses elasticity with time and eventually undergoes a phase of cardiac remodeling that leads to the premature death. The catecholamines, increased preload, act as hypertrophic factors for heart, and these factors can be inhibited via beta- 1 antagonistic property and nitric oxide potentiating activity of nebivolol. 22,26

The nebivolol is a highly selective beta- 1 antagonist that antagonizes the beta-1 receptors present on the heart along with its nitric oxide inducedvasodilatory effect on the blood vessels. Due to these two properties, nebivolol exerts its pharmacological action. The immediate hemodynamic effects of nebivolol are reduced contractility, ejection fraction, and heart rate but these parameters gradually improve with the time and after few months, contractility, and ejection fraction get improved. These improved hemodynamic effects appear due to the decrease in the preload (end-diastolic volume), total peripheral resistance, and the decrease in the sympathetic activity which leads to cardiac remodeling. ${ }^{27}$

The decrease in the preload enhances the effectiveness of the myocardial fiber shortening during systole and the decrease in the afterload increases the efficacy of ventricles to pump the blood.

This is solely due to its unique nitric oxide-potentiating effect that causes vasodilatation. With nebivolol, these effects are maintained for long-term and hence, cardiovascular hospital admission and all-cause mortality are reduced. ${ }^{19-22,27}$

\section{CLINICAL STUDIES RELATED TO NEBIVOLOL THERAPY IN HEART FAILURE}

\section{Nebivolol therapy in Systolic dysfunction}

In different clinical studies, it was found that nebivolol improves the left ventricular function if taken as a long-term therapy. In one such study aimed at the influence of nebivolol on left ventricular function of the patients with the chronic heart failure, which was a placebo-controlled, double-blind, and randomized trial involving 12 patients with the ejection fraction of $13-39 \%$, they found that nebivolol was very well tolerated and improved the NYHA stage in four patients.

Heart rate decreased and the maximal exercise duration and performance were found to be stable. Ejection fraction got enhanced, from (29.8 \pm $10.66 \%$ to $41.2 \pm 10.53 \%$ ); (calculated probability) $=0.007$ whereas placebo did not improve the ejection fraction. The left ventricular end systolic volume was found to be decreased, from $(56.5 \pm 9.40 \mathrm{ml}$ to $50.2 \pm 9.43 \mathrm{ml})$ in the nebivolol group $(p \leq 0.02)$. This result was found after 3 months of nebivolol therapy (doses were $2.5 \mathrm{mg}$ and $5 \mathrm{mg}, \mathrm{n}=6$ ) or placebo $(n=6) .^{28}$ In many comparative studies with the nebivolol and other beta-blockers like carvedilol, it has been found that nebivolol does improve the LV systolic function and hence improve the Ejection fraction. Karkabackak et al. compared the effects of carvedilol and nebivolol on LV systolic functions in patients with non-ischemic HF. In this study, a total number of 61 symptomatic non-ischemic heart failure patients with low ejection fraction $(\mathrm{EF} \leq 40 \%)$ were taken. The patients were randomized to carvedilol and nebivolol. The patients were evaluated echocardiographically at baseline and 3 to 6 months after the target dose. After 6 months, it was found that Ejection fraction got increased, from (34 $\pm 5 \%$ to $36 \pm 5 \%$ ); $\mathrm{p}<0.01$. $\mathrm{LV}$ diameter was reduced along with decreased Isovolumic Contraction Time (IVCT) and Isovolumic Relaxation Time (IVRT). Ejection time was found to be prolonged with nebivolol. IVCT, IVRT, and LV diameter are the parameters for finding the LV systolic functions. ${ }^{29}$

In another comparative study by Lombardo et al. the effects of nebivolol and carvedilol on the LV function in the patients suffering from HF were assessed. In this study, 70 patients with reduced Ejection fraction $(\leq 40 \%)$ were randomly assigned carvedilol or nebivolol for 6 months. After 6 months of treatment with the carvedilol and nebivolol, patients were assessed clinically and by the hematological (particularly, N-terminal pro-brain natri-uretic peptide) and biochemical investigation. 
Besides, ECG (Electrocardiogram), 6-minute walk test (6 MWT), and ventilatory functions were also analyzed. They found that in case of nebivolol, Ejection fraction was improved from $(34 \% \pm 7 \%$ to $38 \% \pm 10 \%)$. The end systolic volume was also found to be reduced from $(72 \pm 35 \mathrm{~mL}$ to $66 \pm 32 \mathrm{~mL})$. A small reduction in the mean NYHA functional class from the baseline was found to be from $(2.3 \pm 0.4$ to $2.2 \pm 0.5)$. There was an increase in walking distance in both the groups. 6-minute walk test showed an increase in the walking distance in both of the carvedilol and nebivolol, no significant variations were seen in case of hematological findings and respiratory functions with both the carvedilol and nebivolol. So, nebivolol was found to be as effective as carvedilol in the study. ${ }^{30}$

In the SENIOR (study of the effects of nebivolol intervention on outcomes and re-hospitalization in the seniors) trial, that was meant to assess the effects of nebivolol on the mortality and cardiovascular hospital admission in the elderly patients with the heart failure and who were $\geq 70$ years. The study included 2128 patients who had a history of heart failure (got hospitalized for the heart failure in the previous year or had the known ejection fraction of $\leq 35 \%$ ). Nebivolol was given to a total of 1067 (initially from $1.25 \mathrm{mg}$ and titrated up to $10 \mathrm{mg}$ once daily) patients whereas placebo was given to 1061 patients. The primary endpoint was a composite of all-cause mortality or cardiovascular hospital admission. The death occurred in a total of $169(15.8 \%)$ on nebivolol therapy and 192 (18.1\%) on placebo $(\mathrm{HR}=0.88,95 \% \mathrm{CI} 0.71-1.08 ; \mathrm{P}=0.21)$, where $\mathrm{HR}$ is Hazard ratio, CI is Confidence Interval, and $\mathrm{P}$ is Calculated Probability. The conclusion drawn was nebivolol is an effective and well-tolerated drug for the treatment of heart failure in elderly. This was the study that involved the elder which contrasts with other studies in this aspect. ${ }^{9,31}$ In another study by Karabacak et al. nebivolol was found to reduce the oxidative stress like that of carvedilol in the patients with non-ischemic heart failure. ${ }^{32}$

There are so many other studies besides these studies that have proven the beneficial effects of nebivolol in heart failure with systolic dysfunction. It is noteworthy that nebivolol and carvedilol both possess vasodilatory effects. However, the mechanism of action of carvedilol presents higher blocking affinity for beta-2 receptors than beta-1 receptor on the heart. This may lead to the consequences or the side effects due to beta- 2 receptor blockade which proves the superiority of nebivolol.

\section{Nebivolol therapy in Diastolic dysfunction}

Most of the clinical trials have been found to be associated with the effects of nebivolol in the patients with reduced ejection fraction or the heart failure with systolic dysfunction and not with the patients of heart failure with preserved ejection fraction or the diastolic dysfunction. However, there are some few numbers of studies that have demonstrated both positive and negative results in heart failure with diastolic dysfunction. In a study, nebivolol (5 mg) was found to decrease the Pre-Ejection Period (PEP) and Pre-Ejection Period/ Left Ventricular Ejection Time (PEP/ LVET) ratio while a traditional blocker, metoprolol, was not found to decrease these parameters.

By decreasing PEP and PEP/LVET ratio, nebivolol showed higher diastolic peak filling rates or improved relaxation in diastolic dysfunction. This was a study which showed positive effects in diastolic dysfunction. ${ }^{33-35}$

Another important contradicting study that showed less significant effects is the ELANDD (Effects of the long-term administration of nebivolol on the clinical symptoms, exercise capacity, and left ventricular function of patients with diastolic dysfunction). In this study, 116 subjects with diastolic heart failure or with preserved ejection fraction, who were in the NYHA functional class II-III, whom left ventricular ejection fraction was $>45 \%$ and who showed echo-doppler signs of LV diastolic dysfunction, were randomized to placebo or nebivolol treatment for 6 months. The primary end point was change in 6 min walk test distance
(6 MWTD) after 6 months. Nebivolol was not found to improve the 6 MWTD efficiently (from $420 \pm 143$ to $428 \pm 141 \mathrm{~m}$ ) and with placebo (from $412 \pm 123$ to $446 \pm 119 \mathrm{~m}$ ). The nebivolol was not found improve the exercise capacity in this study. The Quality of Life improvement by using Minnesota Living with Heart Failure and NYHA class were improved to the same extent in both the groups. ${ }^{36}$ Currently, there are a lot more clinical studies and data are required to verify the fact that nebivolol can improve and boost the diastolic function in parallel to systolic function.

\section{CLINICAL PHARMACOKINETICS}

Following the oral administration of a standard $5 \mathrm{mg}$, nebivolol is absorbed fast and reaches the peak plasma concentration within 1.5-4 hr. The plasma protein binding is specifically with albumin, approximately $98 \%$. The food has minimal effect on the drug absorption, so less regard is given to meals while administering orally. ${ }^{37}$ It is metabolized in the liver primarily by hydroxylation which is a type of oxidation reaction besides oxidative deamination, $\mathrm{N}$ or $\mathrm{O}$-dealkylation, oxygenation at $\mathrm{N}$, $\mathrm{C}$ or $\mathrm{S}$ atoms, etc. This hydroxylation produces active and inactive metabolites. ${ }^{38}$

The important monooxygenase that carries the oxidation reaction that is hydroxylation is cytochrome (CY) P450 isoenzyme 2D6. It is the genetic polymorphism of CYP2D6 that makes the individual 'poor' metabolizer or the 'extensive' metabolizer. The poor metabolizer has very good oral bioavailability (98\%) while the extensive metabolizers have an oral bioavailability of $12 \%$. In poor metabolizers, the main pathway for metabolizing the nebivolol is the glucuronidation, alicyclic monooxidation following the glucuronidation and $\mathrm{N}$-dealkylation. In the extensive metabolizers, mainly hydroxylation in combination with $\mathrm{N}$-dealkylation, alicyclic monooxidation and glucuronidation following the aromatic hydroxylation, and glucuronic acid conjugation of unchanged nebivolol are the major metabolic pathways. So, it is substantial to take CYP2D6 inducer or inhibitor into consideration while giving the nebivolol. For example, CYP2D6 inhibitor fluoxetine increased the peak plasma concentration of the nebivolol three times as compared to the normal peak plasma concentration.

The half-life of nebivolol is more in poor metabolizer and vice versa that is approximately $10 \mathrm{~h}$ in extensive metabolizers and 30-50 hr in poor metabolizer was reported. ${ }^{37,39}$ It was found while reviewing the articles that clinical response and safety profiles in both types of metabolizers are same.

So, dose adjustment in case of poor metabolizers is not needed. The average volume of distribution of nebivolol is $10 \mathrm{~L} / \mathrm{Kg}$ and patient weight seems to have no effect on the volume of distribution. The major route of excretion is through urine and feces. ${ }^{37}$ It is excreted mostly as metabolites; there is $<0.5 \%$ of the unchanged drug that is excreted. Articles pertaining to the pharmacology of nebivolol reported that poor metabolizers excrete about $67 \%$ of nebivolol metabolites in urine and about $13 \%$ in feces. The data of extensive metabolizers was $38 \%$ in urine and $44 \%$ in feces. ${ }^{40}$

\section{ADVERSE EFFECTS AND TOLERABILITY}

Treatment with the nebivolol is usually for the long time duration. In many clinical studies, it has been found that nebivolol is well tolerated and safe. However, there are some different adverse effects that are attributable to the nebivolol, but the most common adverse drug reactions reported are nausea, headache, bradycardia, fatigue, abdominal pain, chest pain, peripheral edema, dizziness, insomnia, dyspnea, and rash. Less incident adverse drug effects include asthenia, hypercholesterolemia, and paresthesia. Some of the adverse effects like bradycardia arise due 
to an increase in the dose and is certified from the pharmacology of the drug. So, overall, tolerability and patient safety are ensured with nebivolol. ${ }^{41}$

\section{DRUG-DRUG INTERACTIONS}

When it comes to interactions, then special caution must be taken when given with CYP2D6 inhibitors like fluoxetine, quinidine or with the CYP2D6 inducers like ethanol, phenytoin, etc. Drug interactions with the nebivolol found in different studies with some commonly administered drugs are given below:

Fluoxetine: It is very important to evaluate the drug interactions with this commonly prescribed anti-depressant due to its potent CYP2D6 inhibiting nature. In the study by Lindamood et al. it was found that when nebivolol $10 \mathrm{mg}$ once daily was co-administered with fluoxetine $20 \mathrm{mg}$ once daily, the plasma concentration of nebivolol got increased. Steady state level of fluoxetine decreased the apparent clearance of nebivolol. This led to the increased value of $\mathrm{C}_{\max }$ and $\mathrm{AUC}_{0-\infty}$, which were 2.3 fold and 6 fold more than when it was not co-administered with nebivolol.

Furosemide: No significant changes in the pharmacokinetic parameters were noted when it was co-administered in a single dose of $40 \mathrm{mg}$ with nebivolol in a single dose of $10 \mathrm{mg}$. The $\mathrm{C}_{\mathrm{ss}}, \mathrm{AUC}_{\tau}$, and CL/F (apparent clearance) of D- nebivolol or L-nebivolol were not changed by furosemide. No change in $t_{1 / 2}$ of nebivolol in poor metabolizers and $\mathrm{C}_{\max }$ in extensive metabolizers was found.

Nebivolol was also not found to affect the pharmacokinetic parameters of furosemide, $\mathrm{C}_{\max }, \mathrm{AUC}_{\text {last }}, \mathrm{AUC}_{0-\infty}$, and $\mathrm{CL} / \mathrm{F}$ of furosemide were unchanged. The same kinds of observations were noted with hydrochlorothiazide and spironolactone.

Losartan: No clinically significant interactions were noted in poor and extensive metabolizers of nebivolol (10 mg once daily) when given with losartan $50 \mathrm{mg}$ once daily. No safety risk was noted when given concomitantly with nebivolol. The $\mathrm{C}_{\max }, \mathrm{AUC}_{\text {last }}$, and $\mathrm{AUC}_{0-\infty}$ of losartan were only slightly reduced by nebivolol.

Digoxin: For the determination of potential interactions with digoxin, 13 healthy subjects in which there were 12 extensive metabolizers and 1 metabolizer, were evaluated in a trial. The doses of digoxin and nebivolol were $0.25 \mathrm{mg}$ once daily and $10 \mathrm{mg}$ once daily. No significant pharmacokinetic parameter changes in digoxin were found. The ratio of $\mathrm{C}_{\max }$ of digoxin (without nebivolol) to $\mathrm{C}_{\max }$ when given concomitantly with nebivolol, was 1.07 . Only 6 subjects experienced adverse effects that too were mild in severity.

Ramipril: 15 subjects involving three poor metabolizers and 12 extensive metabolizers of the age 19-69 years were evaluated to find the interactions with nebivolol. In this, the dose of ramipril and nebivolol was $5 \mathrm{mg}$ once daily and $10 \mathrm{mg}$ once daily. No significant changes in pharmacokinetic parameters were observed. Furthermore, no treatment differences were noted when ramipril was co-administered with nebivolol or alone. 58 adverse drug reactions were noted in a total of 10 subjects, among which fatigue and headache were most common. No serious adverse drug reactions were noted.

Cimetidine and Ranitidine: A randomized placebo-controlled cross-over study was conducted on 12 healthy volunteers to find out the effects of $\mathrm{H} 2$ receptor antagonist on the pharmacokinetics and pharmacodynamics of nebivolol. Each subject was given the placebo, cimetidine (400 mg two times a day), and ranitidine (150 mg two times a day). It was found that ranitidine had no significant effect on the pharmacokinetics of nebivolol and cimetidine increased the $\mathrm{C}_{\max }$ of nebivolol by $21-23 \%$. No clinically significant changes in the pharmacodynamics of the nebivolol were found. ${ }^{8,42}$

\section{FUTURE PROSPECTS WITH NEBIVOLOL}

The drug is approved for the treatment of hypertension and heart failure in Europe. At present, nebivolol is not a US Food and Drugs Administration (FDA) approved drug for the treatment of heart failure although it has been approved for the treatment of hypertension by the FDA. ${ }^{7}$ An FDA advisory panel voted unanimously not to recommend approval of nebivolol for the treatment of chronic heart failure.

The most important reason for this was the weak showing of nebivolol, unmet clinical findings in the SENIOR trial which could not convince FDA. In fact, the SENIOR trial was the only trial that included the patients who were $\geq 70$ years of age and result of the trial also showed benefiting for patients with heart failure.

The epidemiological data of heart failure state that heart failure is associated with morbidity, mortality, and profound health expenditures in the age of 65 or more. So, the primary endpoint of the trial was a composite of all-cause mortality and reduced cardiovascular hospitalization after 3 months of treatment with nebivolol.

There were potential selection and time bias on the basis of which nebivolol could not get the approval from the FDA. The selection bias was homogeneity in the race that means the trial mostly included the white people. There were differences in the population prevalence, and the treatment effects on the basis of race which were found in other previous studies and lastly, the other selection bias in the study was the inclusion of lower-risk population of heart failure or the "healthiest of the elderly" which limited and affected the trial's ability to show the mortality differences between the placebo and nebivolol group. The time duration of 3 months was a potential source of time bias. So, Cardiovascular and Renal Drugs Advisory Committee advised increasing the duration of 3 months to 1 year to enlarge the number of mortality endpoints. ${ }^{43}$ The FDA approved drug for the treatment of heart failure is metoprolol, bisoprolol, and carvedilol. One can expect more trials related to the nebivolol in the elderly and expect that in the future, nebivolol will surely get the approval from the FDA.

\section{CONCLUSION}

Nebivolol is a kind of drug having highest selectivity for the beta-1 receptors than any other beta-blocker. It is being used for the treatment of heart failure as well as hypertension in European countries but till date due to the absence of satisfactory clinical findings, it has not got approval from US FDA. When it comes to pharmacological actions, then it is no less than any other US FDA approved drug like carvedilol. It has wonderful nitric oxide potentiating action by activating the beta- 3 receptors. Nitric oxide has excellent effects on the heart as well as vascular system, for instance, nebivolol protects against myocardial ischemiareperfusion injury, vasodilatation and many more. The most deleterious compensatory mechanisms like cardiac remodeling found in case of heart failure are abated by nitric oxide which can be fatal. Nitric oxide reduces the oxidative injury moderated by alkyl-hydro peroxides, hydrogen peroxides, and xanthine oxidases. Nebivolol is extensively metabolized in the liver by the CYPD26 and there are individual variations in the activity of CYP2D6 that makes the person extensive or poor metabolizer. The adverse effects that are related to nebivolol are not serious and possess good tolerability and safety. With nebivolol, one can be assured to be benefited and if not, then, at least no serious adverse effect and this is the quality of nebivolol which other approved beta blockers do not confer, in other words, benefits are more and risks are less.

Overall, whatever the clinical data that have been found for nebivolol are favorable and we can expect with certainty that if more numbers of clinical trials data are available, nebivolol will get the approval and it will be no 
more side-lined for the therapy of heart failure by the FDA like other beta-blockers currently in use for this disease.

\section{ACKNOWLEDGEMENT}

We are sincerely thankful to our Pharmacology department of our college for guiding us while writing the review article.

\section{CONFLICT OF INTEREST}

The authors declare no conflict of interest.

\section{REFERENCES}

1. Inamdar AA, Inamdar AC. Heart Failure: Diagnosis, Management and Utilization. J Clin Med. 2016;5(7):62. doi: https://dx.doi.org/10.3390\%2Fjcm5070062.

2. Dassanayaka S, Jones SP. Recent developments in heart failure. Circ Res. 2015;117(7):58-63. doi: https://dx.doi.org/10.1161\%2FCIRCRESAHA. 115.305765.

3. Goto T, Wakami K, Fukuta H, Fujita H, Tani T, Ohte N. Patients with left ventricular ejection fraction greater than $58 \%$ have fewer incidences of future acute decompensated heart failure admission and all-cause mortality. Heart Vessels. 2016;31(5):734-43. doi: https://dx.doi.org/10.1007\%2Fs00380-015-0657-1.

4. Fisker FY, Grimm D, Wehland M. Third-generation beta-adrenoceptor antagonists in the treatment of hypertension and heart failure. Basic Clin Pharmaco Toxicol. 2015;117(1):5-14. doi: https://doi.org/10.1111/bcpt.12396.

5. Hobbs R, Boyl A. Heart Failure [Internet]. Cleveland Clinic. 2014. [cited 2018 Feb 11]. Available from: http://www.clevelandclinicmeded.com/medicalpubs/ diseasemanagement/cardiology/heart-failure.

6. Nemichand SK, Laxman SD. Solubility Enhancement of Nebivolol by Micro Emulsion Technique. J Young Pharm. 2016;8(4):356-7. doi: http://dx.doi. org/10.5530/jyp.2016.4.11.

7. Veverka A, Salinas JL. Nebivolol in the treatment of chronic heart failure. Vasc Health Risk Manag. 2007;3(5):647-54.

8. Lindamood C, Ortiz S, Shaw A, Rackley R, Gorski JC. Effects of commonly administered agents and genetics on nebivolol pharmacokinetics: Drug-drug interaction studies. J Clin Pharmacol. 2011;51(4):575-85. doi: https://doi. org/10.1177/0091270010370846

9. Flather MD, Shibata MC, Coats AJ, Parkhomenko A, Borbola J, Cohen A, et al. Randomized trial to determine the effect of nebivolol on mortality and cardiovascular hospital admission in elderly patients with heart failure (SENIORS). Eur Heart J. 2005;26(3):215-25. doi: https://doi.org/10.1093/eurheartj/ehi115.

10. Fongemie J, Felix-Getzik E. A Review of Nebivolol Pharmacology and Clinical Evidence. Drugs. 2015;75(12):1349-71. doi: https://doi.org/10.1007/s40265-0150435-5.

11. Conraads VM, Metra M, Kamp O, De Keulenaer GW, Pieske B, Zamorano J, et al. Eur J Heart Fail. 2012;14(2):219-25. doi: https://doi.org/10.1093/eurjhf/hfr161.

12. Eduardo Toblli J, Digennaro F, Fernando Giani J, Pablo Dominici F. Nebivolol: Impact on cardiac and endothelial function and clinical utility. Vasc Health Risk Manag. 2012;8(1):151-60. doi: https://doi.org/10.2147NHRM.S20669.

13. Maffei A, Lembo G. Nitric oxide mechanisms of nebivolol. Ther Adv Cardiovasc Dis. 2009;3(4):317-27. doi: https://doi.org/10.1177/1753944709104496.

14. Dery AS, Hamilton LA, Starr JA. Nebivolol for the treatment of heart failure. Am J Health Syst Pharm. 2011;68(10):879-86. doi: https://doi.org/10.2146/ ajhp100309.

15. Mason RP. Nitric oxide mechanisms in the pathogenesis of global risk. J Clin Hypertens. 2006;8(s8):31-40.

16. Cockcroft JR, Chowienczyk PJ, Brett SE, Chen CP, Dupont AG, Van Nueten L, et al. Nebivolol vasodilates human forearm vasculature: evidence for an L-arginine/ NO-dependent mechanism. J Pharmacol Exp Ther. 1995;274(3):1067-71.

17. Gauthier C, Tavernier G, Charpentier F, Langin D, Le Marec H. Functional beta3adrenoceptor in the human heart. J Clin Invest. 1996;98(2):556-62. doi: https:// dx.doi.org/10.1172\%2FJCl118823.

18. Gupta Y, Shrivastava A, Duggal D, Patel A, Agrawal S. A New RP-HPLC Method for Simultaneous Estimation of Nebivolol Hydrochloride and Hydrochlorthiazide in Dosage Forms. J Young Pharm. 2009;1(3):264-9. doi: http://dx.doi.org/ 10.4103/0975-1483.57078.

19. Dubois-Rande JL, Zelinsky R, Roudot F, Chabrier PE, Castaigne A, Geschwind H, et al. Effects of infusion of L-arginine into the left anterior descending coronary artery on acetylcholine-induced vasoconstriction of human atheromatous coronary arteries. Am J Cardiol. 1992;70(15):1269-75.

20. James F, Spann JF. Heart failure and ventricular hypertrophy: Altered cardiac contractility and compensatory mechanisms. Am J Cardiol. 1969;23(4):504-10. doi: https://doi.org/10.1016/0002-9149(69)90003-4

21. Wollert KC, Drexler $\mathrm{H}$. Regulation of cardiac remodeling by nitric oxide: focus on cardiac myocyte hypertrophy and apoptosis. Heart Fail Rev. 2002;7(4):317-25.

22. Garcia JA, Incerpi EK. Factors and mechanisms involved in left ventricular hypertrophy and the anti-hypertrophic role of nitric oxide. Arq Bras Cardiol. 2008;90(6):443-50. doi: http://dx.doi.org/10.1590/S0066-782X2008000600010.

23. Aragon JP, Condit ME, Bhushan S, Predmore BL, Patel SS, Grinsfelder DB, et al. Beta3-adrenoreceptor stimulation ameliorates myocardial ischemia-reperfusion injury via endothelial nitric oxide synthase and neuronal nitric oxide synthase activation. J Am Coll Cardiol. 2011;58(25):2683-91. doi: https://doi.org/10.1016/j. jacc.2011.09.033.

24. Wink DA, Hanbauer I, Krishna MC, DeGraff W, Gamson J, Mitchell JB. Nitric oxide protects against cellular damage and cytotoxicity from reactive oxygen species. Proc Natl Acad Sci USA. 1993;90(21):9813-7.

25. Ramanath KV, Balaji DBSS, Nagakishore $\mathrm{CH}$, Mahesh Kumar S, Bhanuprakash M. A Study on Impact of Clinical Pharmacist Interventions on Medication Adherence and Quality of Life in Rural Hypertensive Patients. J Young Pharm. 2012;4(2):95-100. doi: http://dx.doi.org/10.4103/0975-1483.96623.

26. Jackson G, Gibbs CR, Davies MK, Lip GY. ABC of heart failure: Pathophysiology. BMJ. 2000;320(7228):167-70

27. Gauthier C, Tavernier G, Charpentier F, Langin D, Le Marec H. Functional $\beta 3$-adrenoceptor in the human heart. J Clin Invest. 1996;98(2):556-62.

28. Wolf SC, Buck-Muller N, Gorner S, et al. Influence of the beta-blocker nebivolol on the left ventricular function in patients with chronic heart failure. Med Klin. 2003;98(1):1-6.

29. Karabacak M, Dogan A, Tayyar S, Ozaydın M, Erdogan D. Carvedilol and nebivolo improve left ventricular systolic functions in patients with non-ischemic heart failure. Anatol J Cardiol. 2015;15(4):271-6. doi: https://doi.org/10.5152/ akd.2014.5337.

30. Lombardo R, Reina C, Abrignani M, Rizzo P, Braschi A, De Castro S. Effects of nebivolol versus carvedilol on left ventricular function in patients with chronic heart failure and reduced left ventricular systolic function. Am J Cardiovas Drugs. 2006;6(4):259-63. doi: https://doi.org/10.2165/00129784-200606040-00006.

31. Solal AC, Kotecha D, Veldhuisen DJ, Babalis D, Bohm M, Coats AJ, et al. Efficacy and safety of nebivolol in elderly heart failure patients with impaired renal function: insights from the SENIORS trial. Eur J Heart Fail. 2009;11(9):872-80. doi: https://dx.doi.org/10.1093\%2Feurjhf\%2Fhfp104

32. Karabacak M, Dogan A, Tayyar S, Bas HA. The effects of carvedilol and nebivolol on oxidative stress status in patients with non-ischaemic heart failure. Kardio Pol. 2015;73(3):201-6. doi: https://doi.org/10.5603/KP.a2014.0190.

33. Rousseau MF, Chapelle F, Van Eyll, Stoleru L, Hager D, Van Nueten L, et al. Medium-term effects of beta-blockade on left ventricular mechanics: A doubleblind, placebo-controlled comparison of nebivolol and atenolol in patients with ischemic left ventricular dysfunction. J Card Fail. 1996;2(1):15-23.

34. De Cree J, Geukens H, Verhaegen H. Noninvasive cardiac hemodynamics of nebivolol. Drug Invest. 1991;3(1):40-50. doi: https://doi.org/10.1007/ BF03258262.

35. Grossman W. Diastolic dysfunction and congestive heart failure. Circulation. 1990;81(2):1-7.

36. Conraads VM, Metra M, Kamp O, Vardas PE, Pieske B, Zamorano J, et al Effects of the long-term administration of nebivolol on the clinical symptoms, exercise capacity, and left ventricular function of patients with diastolic dysfunction: Results of the ELANDD study. Eur J Heart Fail. 2012;14(2):219-25. doi: 10.1093/eurjhf/hfr161.

37. Nodari S, Metra M, Dei CL. Beta-blocker treatment of patients with diastolic heart failure and arterial hypertension. A prospective, randomized, comparison of the long-term effects of atenolol vs. Nebivolol Eur J Heart Fail. 2003;5(5):621-7.

38. Mangrella M, Rossi F, Fici F, Rossi F. Pharmacology of nebivolol. Pharmacol Res. 1998;38(6):419-31. doi: https://doi.org/10.1006/phrs.1998.0387.

39. Hilas O, Ezzo D. Nebivolol (bystolic), A novel Beta blocker for hypertension. PT. 2009;34(4):188-92.

40. Ozaydin M, Yucel H, Kocyigit S, Adali MK, Aksoy F, Kahraman F, et al. Nebivolol versus Carvedilol or Metoprolol in Patients Presenting with Acute Myocardial Infarction Complicated by Left Ventricular Dysfunction. Med Princ Pract. 2016;25(4):316-22. doi: https://doi.org/10.1159/000446184

41. Kamali F, Howes A, Thomas SH, Ford GA, Snoeck E. A pharmacokinetic and pharmacodynamic interaction study between nebivolol and the $\mathrm{H} 2$-recepto antagonists cimetidine and ranitidine. Br J Clin Pharmacol. 1997;43(2):201-4. doi: https://dx.doi.org/10.1046\%2Fj.1365-2125.1997.54212.x.

42. Stiles S. FDA Advisory Panel Votes against Nebivolol Approval for Heart Failure [Internet]. Medscape. 2010. [cited 2018 Feb 11]. Available from: https://www. medscape.com/viewarticle/715059\#vp_2.

43. Sule SS, Frishman W. Nebivolol: new therapy update. Cardiol Rev. 2006; 14:259 64. doi: https://doi.org/10.1097/01.crd.0000223651.03023.8e 